\title{
Reference diameter in calculations of creep strain for steam pipelines
}

\author{
Stanisław Łopata ${ }^{1, *}$ \\ ${ }^{1}$ Cracow University of Technology, al. Jana Pawła II 37, 31864 Kraków, Poland
}

\begin{abstract}
Recommended methods of the operational safety assessment of high-pressure steam pipelines include periodic testing that enables determination of creep strain and the creep rate. The accuracy with which the two quantities are determined depends, among others, on the control of the testing conditions and on the assumed reference diameter. The analysis conducted herein concerns the impact of the reference diameter on the results characterizing the creep phenomenon in the pipeline elements (straight sections and bends). In this respect, the initial ovality of the pipeline cross-section is an important parameter. The calculations are made using own results obtained from many years of the steam pipeline creep testing, including tests performed after the expiry of the computational service life.
\end{abstract}

\section{Introduction}

Thermal cycles realized in commercial and industrial power plants incorporate not only their main components, i.e. boilers, turbines and generators, but also auxiliary equipment including steam pipelines. These may be combined into unit- or collector-type systems which may sometimes be very sophisticated. Their failure usually involves the power unit shutdown even though the boiler and the turbine are in good working order. Due to high values of temperature and pressure of superheated steam transmitted along them, such pipelines are often referred to as high-temperature or high-pressure ones. They are often affected by creep and thermo-mechanical fatigue phenomena which, as their operation time gets longer, change the properties of materials they were made of. The creep process is the dominant one in pipelines, and it manifests itself by an increment in their diameter.

According to the standard [1], the steam pipeline creep can be tested by means of periodic measurements of the diameter on what is referred to as measuring pins. The pins are installed on the external surface of pipes, typically in two directions which are perpendicular to each other and to the pipe axis [2-5]. Although rare in practice, more directions can occasionally be used [6]. The principle that governs the measurement of the steam pipeline creep-related plastic strain is illustrated in Fig. 1 $[4,5]$. It may be added that the presented method belongs to non-destructive testing, which certainly is its advantage.

In the power industry in Poland, periodic testing of creep for steam pipelines is an element of their diagnostics. It is required by regulations of the Technical Inspection Office (UDT) [7] and diagnostic tests have to be performed throughout the pipeline operating lifetime.
In practice, apart from due care, the testing sufficient accuracy is ensured by appropriate identification of conditions in which tests are carried out. This comes down to measuring the temperatures of the pipeline wall and of the micrometer frame, which - as results from many years of in-house experience - usually differ from each other and their values obtained in subsequent tests are different, too [4].

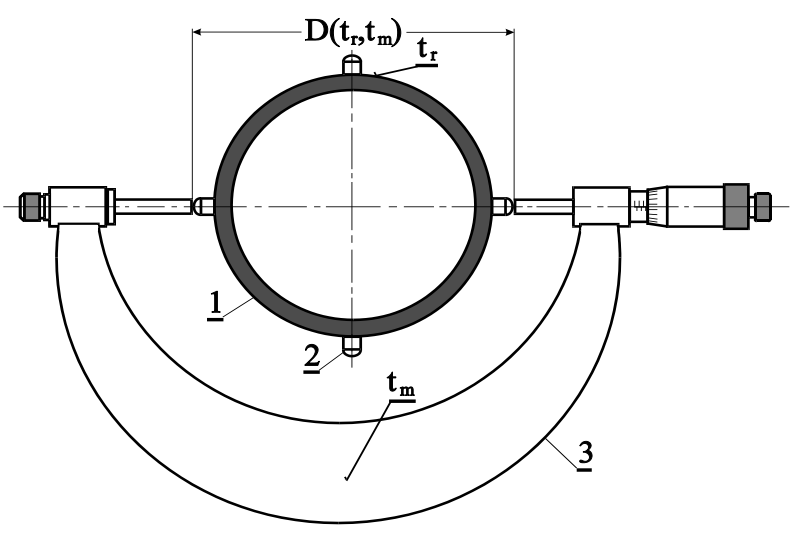

Fig. 1. Location of measuring pins on the steam pipeline and the principle of testing the increment in its outer diameter: 1 pipeline, 2 - measuring pin, 3 - micrometer, $t_{r}, t_{m}$ - temperatures of the pipe wall and of the micrometer frame, respectively.

Knowing temperatures $t_{r}$ and $t_{m}$, it is possible to recalculate the diameter obtained from measurements $D\left(t_{r}, t_{m}\right)$ - Fig. 1 - to its value corresponding to the assumed reference condition, i.e. the temperature of $20^{\circ} \mathrm{C}$. This enables an assessment by comparing the results with the values determined earlier and with those adopted (recognized) as permissible. The recalculation is most often performed using the following relation [2-5]:

*Corresponding author: lopata@mech.pk.edu.pl 


$$
D\left(t_{o}\right)=\frac{D\left(t_{r}, t_{m}\right)+b}{1+\beta_{r}\left(t_{r}-20\right)-\beta_{m}\left(t_{m}-20\right)}
$$

where:

b - micrometer correction (average value of corrections determined prior to and after the measurement), $\mathrm{mm}$,

$D\left(t_{o}\right)$-diameter measured on measuring pins, after recalculation to the reference condition, i.e. temperature $t_{o}=20^{\circ} \mathrm{C}, \mathrm{mm}$,

$D\left(t_{r}, t_{m}\right)$-diameter measured on measuring pins in real conditions of the measurement, i.e. at temperatures $t_{r}$ and $t_{m}, \mathrm{~mm}$,

$t_{m}, t_{r} \quad$-temperature of the micrometer frame and of the pipeline, respectively, ${ }^{\circ} \mathrm{C}$,

$t_{o} \quad$-reference temperature, ${ }^{\circ} \mathrm{C}$,

$\beta_{r}, \beta_{m} \quad$ - linear thermal expansion coefficient of the pipeline and the micrometer material, respectively, $\mathrm{mm} /(\mathrm{mm} \cdot \mathrm{K})$.

The results obtained after different times of the steam pipeline operation make it possible to find quantities that characterize the pipeline creep, i.e. the creep-related plastic strain (or in short: creep strain, as it is often referred to in practice and even in reference literature), and/or the creep rate.

\section{Reference diameter in calculations of the steam pipeline creep strain}

One of the above-mentioned quantities that characterize the pipeline material, taking account of its real operating time, is the creep strain understood as circumferential strain and defined by the following relation (for the outer generating line):

$$
\varepsilon=\frac{\Delta O_{z}}{O_{z}} \cdot 100 \%=\frac{\Delta D_{z}}{D_{z}} \cdot 100 \%
$$

where:

$D_{z} \quad$ - pipeline outer diameter (according to the measurement made prior to the pipeline commissioning), $\mathrm{mm}$,

$O_{z} \quad-$ pipeline outer perimeter (according to the measurement made prior to the pipeline commissioning), $\mathrm{mm}$,

$\Delta D_{z} \quad$-increment in the pipeline outer diameter (after $\tau$ of operating hours), $\mathrm{mm}$,

$\Delta O_{z} \quad$-increment in the pipeline outer perimeter (after of operating hours), $\mathrm{mm}$.

The results obtained during the steam pipeline operation from systematic measurements of diameters on the measuring pins make it possible to calculate strain values $\varepsilon=\varepsilon_{i}$, which arise in the time between subsequent measurements (where $i$ denotes the measurement number), using the following equation:

$$
\varepsilon=\varepsilon_{i}=\frac{D_{i}-D_{1}}{D_{z}} \cdot 100 \%
$$

where:

$D_{1}, D_{i} \quad$ - diameter on measuring pins, recalculated to the reference condition, for the first and the $i$ th measurement (measurements performed before the pipeline commissioning and after time $\tau$ of operating hours, respectively), mm,

$D_{z} \quad$ - pipeline outer diameter (according to the measurement made prior to the pipeline commissioning), $\mathrm{mm}$,

$\varepsilon \quad-$ pipeline creep strain, $\%$,

$\varepsilon_{i} \quad-$ steam pipeline creep strain obtained after time $\tau$ of operating hours, $\%$.

As it was already mentioned, the pipeline strain measurements are performed in two directions perpendicular to each other. In this situation relation (2) is correct assuming that the pipe has the shape of a perfect circle. The increment in the diameter is then independent of the measurement direction. In reality, however, this is not the case because pipelines are characterized by their cross-section ovality [4, 5, 8, 9], which means that the value of diameter $D_{z}$ is different in each direction. Consequently, using relation (3) and depending on the assumed definition of this quantity, i.e. $D_{z}$ (to which the increase of diameter measured on the measuring pins is referred), the obtained values of $\varepsilon_{i}$ will differ from each other. This will naturally concern both the values calculated based on the data for each measurement direction and for the average ones. The latter can be achieved by averaging the final results obtained earlier for each of the two "directions" independently or by prior averaging of quantities $D_{i}, D_{1}$ and $D_{z}$ appearing in relation (3). Therefore, the computational relations for the two possible methods will be as follows:

- in the first method, which is more common in the power engineering practice in Poland, it is assumed that $D_{z}$ is the average of the values obtained for the two directions [2-5, 10], and the increments determined in both directions are related thereto (by the following equations):

$$
\begin{gathered}
D_{z(s r)}=0.5\left(D_{z(1)}+D_{z(2)}\right) \\
\varepsilon_{1}=\varepsilon_{i(1)}=\frac{D_{i(1)}-D_{1(1)}}{D_{z(s r)}} \cdot 100 \% \\
\varepsilon_{2}=\varepsilon_{i(2)}=\frac{D_{i(2)}-D_{1(2)}}{D_{z(s r)}} \cdot 100 \% \\
\varepsilon=\varepsilon_{i(s r)}=0.5\left(\varepsilon_{i(1)}+\varepsilon_{i(2)}\right)
\end{gathered}
$$

or (if for each of the measurement directions the quantities measured on the measuring pins are also averaged first):

$$
\begin{aligned}
& D_{i(s r)}=0.5\left(D_{i(1)}+D_{i(2)}\right) \\
& D_{1(s r)}=0.5\left(D_{1(1)}+D_{1(2)}\right)
\end{aligned}
$$




$$
\varepsilon=\varepsilon_{i(s r)}=\frac{D_{i(s r)}-D_{1(s r)}}{D_{z(s r)}} \cdot 100 \%
$$

- in the second method, in every case $D_{z}$ is the value obtained for a given direction of the measurement $[11,12]$ :

$$
\begin{gathered}
\varepsilon_{1}^{\prime}=\varepsilon_{i(1)}^{\prime}=\frac{D_{i(1)}-D_{1(1)}}{D_{z(1)}} \cdot 100 \% \\
\varepsilon_{2}^{\prime}=\varepsilon_{i(2)}^{\prime}=\frac{D_{i(2)}-D_{1(2)}}{D_{z(2)}} \cdot 100 \% \\
\varepsilon^{\prime}=\varepsilon_{i(\mathrm{~s} r)}^{\prime}=0.5\left(\varepsilon_{i(1)}^{\prime}+\varepsilon_{i(2)}^{\prime}\right)
\end{gathered}
$$

where (in equations (4) - (11)):

$D_{1}, D_{i} \quad$-diameter on measuring pins, recalculated to the reference condition, for the first and the $i$ th measurement (measurements performed before the pipeline commissioning and after time $\tau$ of operating hours, respectively), $\mathrm{mm}$,

$D_{z} \quad$ - pipeline outer diameter (according to the measurement made prior to the pipeline commissioning), $\mathrm{mm}$,

$D_{z(1)}, \quad-$ pipeline outer diameter in direction (1) and

$D_{z(2)} \quad$ (2), respectively (the directions are perpendicular to each other), $\mathrm{mm}$

$\varepsilon, \varepsilon^{\prime} \quad-$ pipeline creep strain, \%,

$\varepsilon_{i} \quad-$ steam pipeline creep strain obtained after time $\tau$ of operating hours, $\%$.

In equations (4)-(11), in nomenclatures with the meaning as defined in the marking list above, subscripts $s r, 1$ and 2 (in parentheses) correspond to the following values, respectively: the average one and the ones in the two assumed directions -1 and 2 - which are perpendicular to each other (for horizontal pipelines it may be assumed that the directions will be in the vertical and in the horizon planes, and for vertical ones - they will be assumed in relation to e.g. the building longitudinal axis, i.e. the vertical or the horizontal direction thereto).

It should be noted that formulae (6) and (9) are equivalent. Consequently, they will give identical values. It may also be added that:

- sometimes it is not the pipeline initial diameter (i.e. the one measured prior to the pipeline commissioning) that is assumed as the reference value, but the average determined based on the two diameters measured on the measuring pins during the first measurement (recalculated for the reference temperature) and adopted as the reference measurement [3]. In such cases, the general structure of the formulae corresponds to relations (5a), (5b) and (9), respectively, where $D_{z(s r)}$ should be replaced by $D_{1(s r)}$. However, this approach is inappropriate because the dimension additionally takes account of the height of both measuring pins;
- in some cases, the pipeline nominal diameter was assumed as the reference parameter;

- as a basis for the calculation of creep strain $\varepsilon_{i}$, measurement results obtained after a certain time of the pipeline operation, e.g. after $5000 \div 8000 \mathrm{~h}$, were also used [3] instead of those obtained prior to the pipeline commissioning (in this method the assumption is that the pipeline material is characterized by the second stage of the creep process). Such an approach is also inappropriate because in this situation the values calculated based on the data obtained from subsequent measurements are in fact strains that exceed a certain strain value obtained prior to such a reference measurement. While assessing creep, it is not right to omit this ,initial" value. It should also be noted that such a „delay" is not equivalent to the steam pipeline material entering the second stage of the creep process $[4,5,13-15]$. Strain values obtained in this way are underrated.

The issue of different possible definitions of the diameter to be adopted as the reference quantity in calculations of the steam pipeline creep strain has not been analysed yet (and therefore there is no reference literature on the problem). It should be supposed that its impact on determined values may be negligible at low values of the pipeline cross-section ovality. At higher values, however, this is no longer so obvious. This is due to the fact that the quantity, i.e. the initial ovality (and the pipe wall non-uniform thickness on the perimeter), has a great effect on creep strain values, as proved by earlier analyses of the problem $[9,16,17]$. The initial ovality characterizing the profile of the pipeline crosssection in the place where the measuring pins are installed (Fig. 1) can be determined using the following relation:

$$
a_{p}=\frac{2\left(D_{z w}-D_{z m}\right)}{D_{z w}+D_{z m}} \cdot 100 \%
$$

where:

$a_{p} \quad-$ initial ovality of the pipeline cross-section profile, $\%$,

$D_{z m}, D_{z w}$ - pipeline outer diameter (in a given crosssection, smaller and bigger one, respectively, in directions perpendicular to each other; it may therefore be assumed that e.g. $D_{z m}=D_{z(1)}$ and $D_{z w}=D_{z(2)}, \mathrm{mm}$.

\section{Calculation results and analysis}

Using own results of creep testing of high-pressure steam pipelines obtained during many years of measurements performed in one of the Polish thermal power plants, creep strain was calculated by means of the two methods presented above, i.e. using formulae (4)-(11). Two facilities were selected for the analysis. They are made of the same (Cr-Mo-V) steel grade, operated at similar superheated steam parameters (temperature about $530-535^{\circ} \mathrm{C}$, pressure about 9.2-9.4 
$\mathrm{MPa}$ ) and tested systematically from the time of commissioning. They are straight pipeline sections which differ from each other in initial ovality calculated from relation (12).

The creep strain values (both average ones and those obtained in two different directions) are presented in Figs. 2-5. The figures also include data concerning the tested pipelines, i.e. the pipe nominal dimensions, the operating parameters (for transported superheated steam), the initial diameters (in both measuring directions) and the initial ovality value.

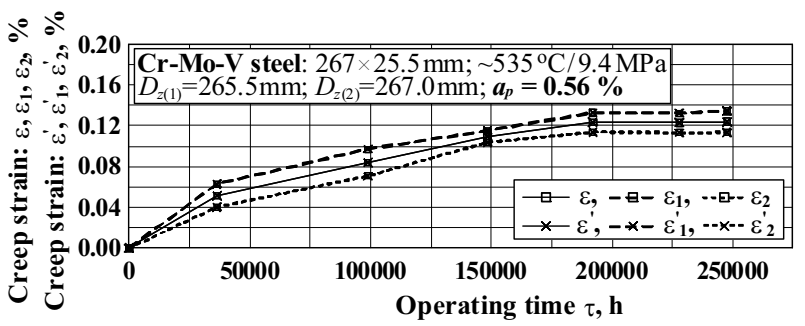

Fig. 2. Pipeline creep curves (for $a_{p}=0.56 \%$ ) plotted according to formulae (4)-(6) and (10a)-(11).

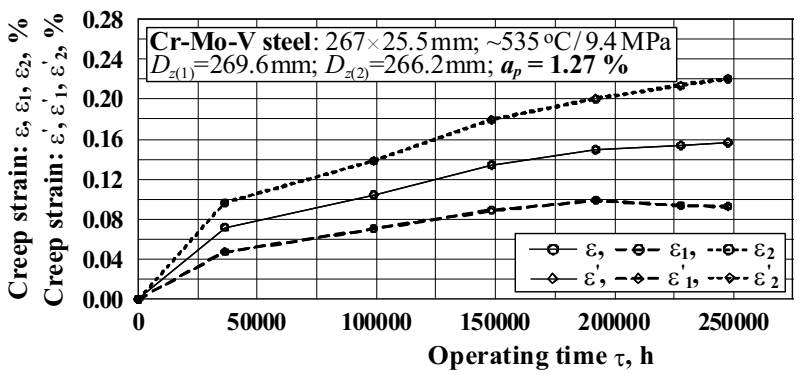

Fig. 3. Pipeline creep curves (for $a_{p}=1.27 \%$ ) plotted according to formulae (4)-(6) and (10a)-(11)

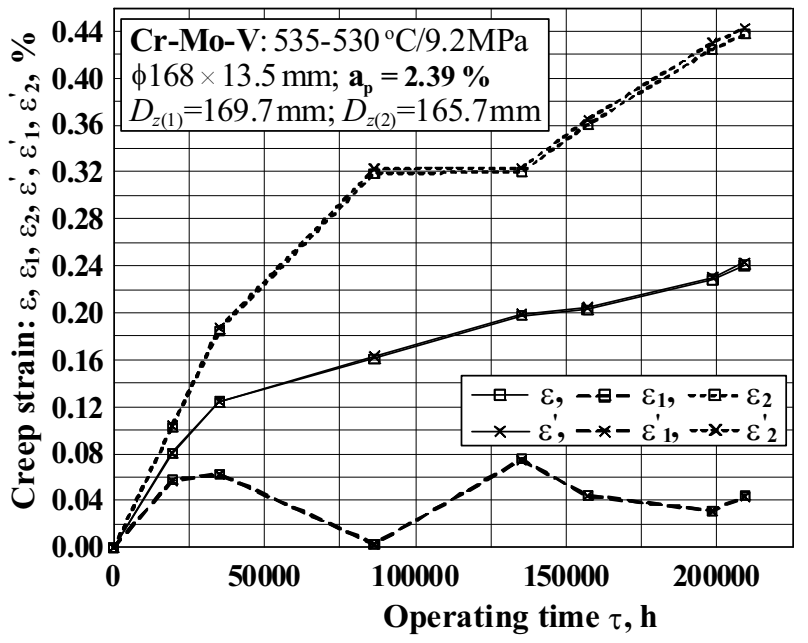

Fig. 4. Pipeline creep curves (for $a_{p}=2.39 \%$ ) plotted according to formulae (4)-(6) and (10a)-(11).

An analysis of Figs. 2-5 indicates that the history of changes in average values of creep strain $\left(\varepsilon, \varepsilon^{\prime}\right)$, i.e. the creep curves, are characteristic of the creep phenomenon [13-15]. One of its features is the slow, time-dependent plastic deformation of the pipeline material (here represented by the above-mentioned increment in the pipe diameter). Two of the phenomenon three stages are visible: the entire first stage (the unsteady-state process taking place at a creep rate continuously decreasing with time until no more changes occur in it) and the second one (the steady-state process with a constant creep rate). It may be added that the last stage of the phenomenon is the accelerated increase in the material deformation (the process occurs with a rise in the creep rate leading to destruction, which makes it undesirable in the power equipment operation - the elements operating in creep conditions should be replaced early enough). All the creep process stages mentioned above are also referred to as first-order (primary) creep, second-order (secondary or steady-state) creep and third-order (tertiary or pre-destruction) creep.

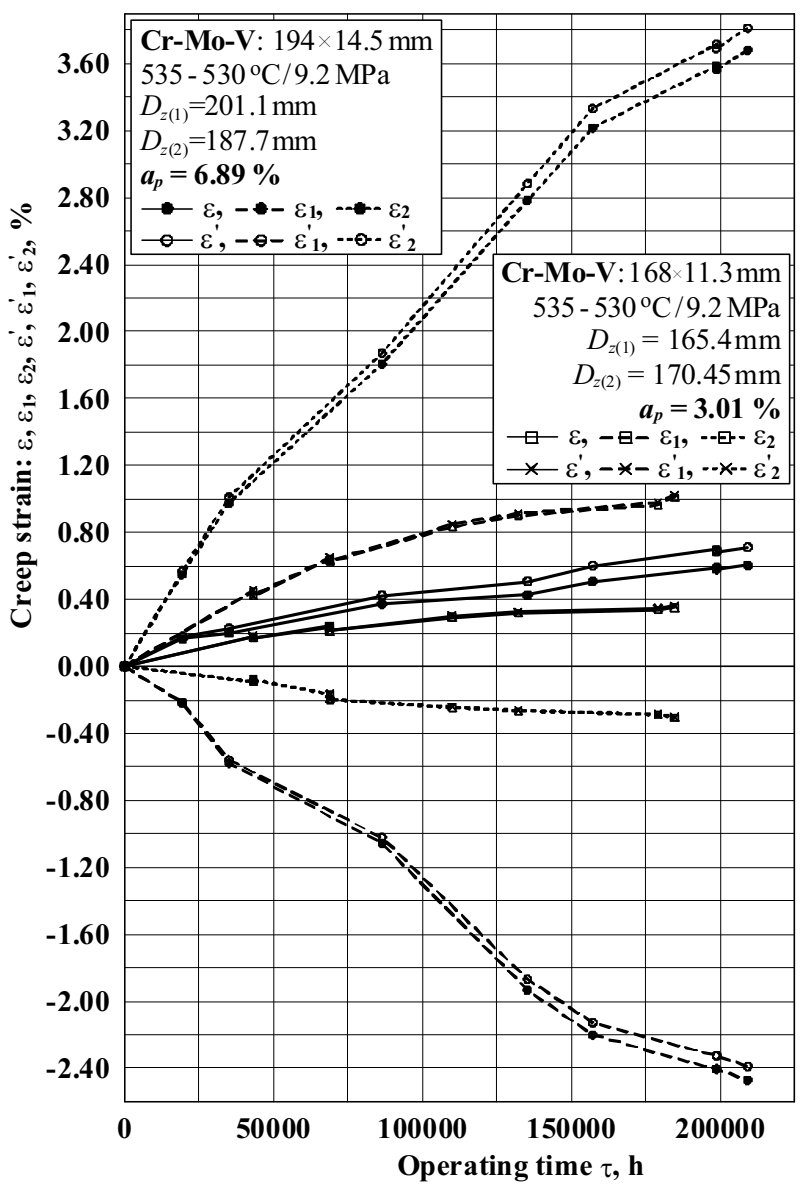

Fig. 5. Pipeline creep curves (for $a_{p}=3.01 \%$ and $a_{p}=6.89 \%$ ) plotted according to formulae (4)-(6) and (10a)-(11)

The above remark has a general nature, but more specific characteristics can be formulated based on Figs. 2-5, such as:

- average values of creep strain in steam pipelines (made of similar materials and with similar operating parameters) are reached after comparable times of operation and they depend on the initial ovality value (the higher it is, the bigger the deformation). For example for the facilities under consideration: for $a_{p}$ of $0.59 \%, 1.27 \%, 2.39 \%, 3.01 \%$ and $6.89 \%$ after 100,000 hours of operation the values of $\varepsilon$ are: $0.084 \%, 0.104 \%, 0.170 \%, 0.280 \%$ and $0.395 \%$, respectively; 
- the curves plotted independently for the two measuring directions are substantially dependent on the initial ovality value. As it rises, the differences between the creep curves, e.g. $\varepsilon_{1}$ and $\varepsilon_{2}$ or $\varepsilon_{1}^{\prime}$ and $\varepsilon_{2}^{\prime}$, get bigger; it can also be seen that for the direction in which the pipeline diameter $\left(D_{z(1)}\right.$ or $\left.D_{z(2)}\right)$ is smaller, the deformations are bigger and vice versa. In the cases under analysis where $a_{p}>3 \%$ (cf. Fig. 5) even "negative" strain values can be observed for the direction in which the pipeline diameter is bigger (and, respectively, high positive values in the other direction). Therefore, it may be stated that with time the pipeline profile gets closer to the round one (its ovality decreases);

- the determined creep strain values (both the average ones and those determined independently for the two measuring directions) are affected by the reference diameter, and the impact gets bigger with a rise in the initial ovality value. Relations (10a)-(11) give higher values compared to relations (4)-(6). However, analysing the average creep strain values (which are reliable in terms of the assessment of the pipeline suitability and operational safety), it should be noted that from the practical point of view the differences between the values obtained by means of relation (6) for $\varepsilon$ and relation (11) for $\varepsilon^{\prime}$ are negligible for low values of the initial ovality. For the facilities under consideration, the relative values of those differences (denoted as $\delta$ ) are shown in Fig. 6; the value is calculated from the following formula:

$$
\delta=\frac{\varepsilon^{\prime}-\varepsilon}{\varepsilon} \cdot 100 \%
$$

where:

$\delta \quad$-relative difference in creep-related strain determined using relations (11) and (6), \%

$\varepsilon, \varepsilon^{\prime} \quad$ - pipeline creep strain, $\%$

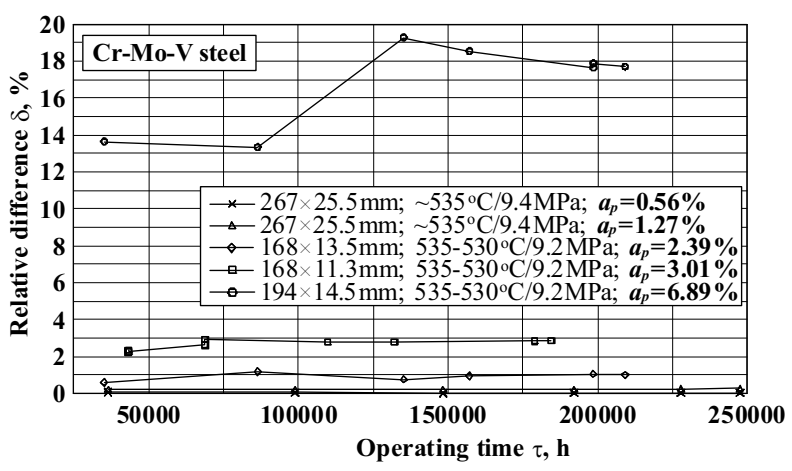

Fig. 6. Relative values of the difference in creep strain determined using relations (4)-(6) and (10a)-(11) for steam pipelines made of similar steel grades, operated at similar parameters of transported superheated steam and characterized by different initial ovality.

Using the determined relative differences in creep strain $\delta$, their average values were calculated in the second stage of the creep process of the analysed pipelines. For certainty it is assumed that in each case the second stage occurred after 75,000 hours of operation. The obtained results are presented as a function of initial ovality in Fig. 7.

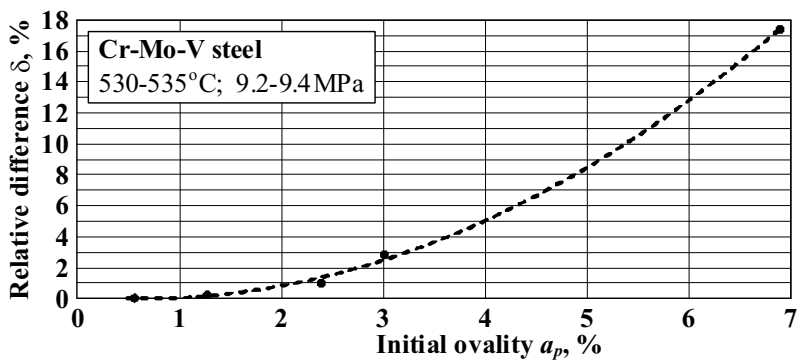

Fig. 7. Relative value of the difference in creep strain determined using relations (4)-(6) and (10a)-(11) depending on initial ovality of the steam pipelines under analysis.

Based on Fig. 7 it can be noticed that the impact of the adopted reference diameter in the calculations of the steam pipeline creep strain is more essential if the pipeline initial ovality is bigger than $2.5 \div 3.0 \%$. Below this limit the differences in the obtained values are relatively small and do not exceed $1.6 \div 2.9 \%$. A rise in initial ovality over 3.0\% involves a "fast" increase in the difference - for $a_{p} \approx 4.5 \div 6.0 \%$ the difference is: $\delta \approx 7 \div 13 \%$, respectively, and in the case of the tested pipeline with ovality $a_{p}=6.89 \%$ it gets as high as $\delta \approx 17.4 \%$.

\section{Concluding remarks}

Determination of parameters characterizing the creep process of materials which are used to make pressure elements of power engineering installations and equipment is an essential task of technical diagnostics. One of the parameters is creep-related plastic strain (usually referred to as creep strain). The strain value, which rises with the time of the steam pipeline operation, is a reliable indicator in the assessment of the pipeline suitability for further operation and of operational safety. For this reason, the accuracy with which the quantity is determined is of great importance, too.

In the calculations of the steam pipeline creep strain, performed independently for each of the two mutually perpendicular directions in which the measuring pins are installed (identical to the directions in which periodic tests are carried out), the reference parameter is the diameter defined prior to the pipeline commissioning. The diameter can be determined as an average value of diameters measured in the two directions (which means that for both directions it is the same) or it can be the diameter corresponding to one specific direction only. Due to that, the creep strain calculated in one specific direction has different values. Consequently, the average values obtained based on data from the two testing directions are also different. The differences are strongly affected by the tested pipeline initial ovality (profile) and they get bigger with a rise in it.

Practically, the steam pipeline creep strain calculation results obtained by means of the methods presented above differ only slightly, i.e. they do not 
exceed $3 \%$ provided that the pipe initial ovality is smaller than $3.0 \%$, which means that it does not matter which method is actually applied. In the case of profiles with initial ovality higher than $3.0 \%$, the differences are substantially bigger (e.g. for ovality of $4.5,6.0$ and $6.9 \%$ they total about 7, 13 and $17 \%$, respectively, and it can be seen that the rate of the rise is characteristically „fast"). Therefore, it is recommended that the method described by formulae (10a)-(11) should be used because it gives higher results, thus ensuring higher safety in terms of the performed assessment of the pipeline suitability for further operation (especially in periods extended beyond the computational operating lifetime). The results of periodic creep strain testing are compared with permissible values and the steam pipeline diagnostics based on this particular method offers a wider safety margin.

\section{References}

1. EN 45510-7-1:1999. Guide for procurement of power station plant, equipment and systems - Part 7-1: Pipework and valves - High pressure piping systems

2. F. Młynarski, Changes in steel properties of the boiler equipment with high steam parameters (in polish), Cracow University of Technology, Zeszyt Naukowy No 12, series: Mechanika No 11 (1963)

3. M. Drop, H. Kruczalak, E. Włoczyk, Guidelines for plastic strain testing - creep measurements (in polish), ZP-BE „Energopomiar”, Gliwice (1977)

4. S. Łopata, Lifetime of high-pressure steam pipelines (in polish), Zeszyty Naukowe Politechniki Krakowskiej, series: Mechanika No 81, Kraków (2001)

5. S. Łopata, Proceedings of the Ninth International Conference on Pressure Vessel Technology, 2, p. 621, Sydney, Australia (2000)

6. G. Thoraval, Nuclear Engineering and Design 116, North-Holland, Amsterdam (1989)

7. Technical Inspection Office, Technical conditions of technical inspection. Pressure equipment. Steam pipelines connecting the boiler to the turbine (in polish) TD-UC-90/RC, Warszawa (1991)

8. S. Łopata, Zeszyty Problemowe. Badania nieniszczące, No 2, pp. 77-82, Warszawa (1997)

9. S. Łopata, M. Augustyniak, K. Kwintowski, Proceedings of the Sixth International Congress on Thermal Stresses, 1, pp. 409-412, Austria (2005)

10. W. Bęczkowski, Power pipelines (in polish), WNT, Warszawa (1963)

11. Steel for boilers and organization of control over their behavior in the high steam parameters. Installation and operation of high-pressure boilers (in russian), Ed. by S.C. Faerman, S.M. Schuher, GEI, Moscow, Leningrad (1949)

12. N.F. Osipowski: High-pressure boilers (in polish), PWT, Warszawa (1956)

13. F. Garofalo, Fundamentals of Creep and Creep Rupture in Metals. The Macmillan Company, New York, Collier-Macmillan Limited, London (1961)
14. M.F. Ashby, D.R.H. Jones, Engineering Materials. An Introduction to their Properties and Applications. Butterworth-Heinemann Ltd. (1993)

15. A. Jakowluk, Creep and fatigue processes in materials (in polish), WNT, Warszawa (1993)

16. S. Łopata, Creep of the steam pipeline with wall thickness non-uniformity, Advanced Materials Research, 875-877, pp. 500-506 (2014)

17. S. Łopata, K. Kwintowski, Creep of steam pipelines with ovality cross-section, Czasopismo Techniczne. Mechanika (Technical Transactions, Mechanics), Cracow University of Technology, z. 2-M, pp. 181190 (2008) 